\title{
EL NUEVO PAPEL DE LAS DIPUTACIONES PROVINCIALES
}

\author{
POR \\ LUIS ORTEGA \\ Catedratico de Derecho Administrativo \\ UNIVERSIDAD DE CASTILLA-LA MANCHA.
}

I. UNA COMUNIDAD LOCAL COMUN, UN INTERES LOCAL COMUN.

Tras la Constitución de 1978, las Diputaciones Provinciales han incorporado un conjunto importante de elementos que son producto de un doble resultado: la nueva concepción del régimen local español caracterizado por los principios de la garantía institucional y la autonomía y el nuevo modelo de descentralización política territorial del denominado Estado de las Autonomías.

Estos nuevos elementos han dado lugar a un nuevo sistema de ordenación del régimen local en el cual el papel de las Diputaciones ha sido cuestionado por algunos como elemento superfluo, cuando no ha sido calificado como un escalón innecesario para la buena marcha de los asuntos públicos.

La Constitución, en cualquier caso, parte de reconocer a la Provincia como uno de los ámbitos territoriales en los que se organiza el Estado, caracterizada por constituirse en cuanto que agrupación de $\mathrm{Mu}$ nicipios, y dotada de autonomía para la gestión de sus intereses. De esta definición constitucional debe extraerse la principal consecuencia: en cuanto que agrupación de Municipios, la Provincia constituye por antonomasia una comunidad local $y$, por ende, la naturaleza de sus intereses está estrechamente ligada a la naturaleza de los intereses de los Municipios que la conforman.

A esta configuración constitucional, viene a unirse el sistema electoral que contempla una única instancia directa de representación local: las elecciones municipales, de cuya "agrupación" de resultados se obtiene la representación Provincial. De aquí que sea legitimo afirmar que sólo hay una única comunidad política local y que el nivel local sólo puede contemplarse desde una única perspectiva de intereses locales. En efecto, el papel de la Diputación, como ente de representación democrática derivada de la representación municipal, estriba en mantener dentro del poder local, del poder político derivado de las elecciones municipales, parcelas de actividad supramunicipal que, 
de otro modo, irían a parar a un ente de representación política distinta como son las Comunidades Autónomas. De este modo lo supramunicipal, los intereses implícitos a la agrupación de Municipios, gracias a la instancia provincial no saltan del poder local a otro poder político de ámbito territorial superior. Como ha señalado LEGUINA, la autonomia provincial es una "autonomía de apoyo a los municipios y que solo en función de dicho apoyo tiene justificación".

Esta es la óptica que ha presidido la elaboración de la Ley Reguladora de las Bases del Régimen Local de 1985 (LRBRL). En primer lugar mediante la Provincia, el poder local tiene encomendado en régimen de autogobierno, como fin propio y especifico, garantizar los principios de solidaridad y equilibrio intermunicipales $y$, por tanto es el propio poder local el que asegura la prestación integral y adecuada en la totalidad del territorio provincial de todos los servicios de - competencia municipal (art. 31 LRBRL).

A esta función atribuida a la Provincia se refieren, indudablemente, las competencias que tienen atribuidas como propias las Diputaciones en el artículo $36 \mathrm{del}$ mismo texto legal, las cuales se extienden, como se recordará, a cuatro actividades esenciales:

- Coordinación de los servicios municipales entre sí.

- Asistencia y cooperación, especialmente dirigida a los Municipios de menos capacidad económica y de gestión.

- Prestación de servicios públicos de carácter supramunicipal y, en su caso, supracomarcal.

- Fomento y administración de los intereses peculiares de la Provincia.

La característica principal de este diseño competencial estriba en que las Diputaciones tienen garantizada una función y unos instrumentos para desarrollarla, sin que quede prefigurado directamente el ámbito de actividades materiales en que se concreta su actividad. Sin embargo, estas actividades materiales son fáciles de localizar. Las encontramos en los amplios listados de los artículos 25 y 26 de la LRBRL. Estos ámbitos materiales, por la nueva concepción constitucional de la autonomía local, están descritos en el elenco de materias sobre las cuales los Municipios deben asumir competencias por virtud de la labor del legislador sectorial. Incluso dentro de la propia LRBRL, dentro de los ámbitos materiales en los que se han determinado servicios municipales mínimos, el articulo 26, apartado 3 , prevé expresamente la intervención de la Diputación, mediante la coordinación cooperación o prestación directa del servicio. Ambitos materiales que comprenden:

- Abastecimiento de agua potable.

- Acceso a núcleos de población. 
- Control de alimentos y bebidas.

- Tratamiento de residuos.

- Protección civil.

- Prestación de servicios sociales.

- Instalaciones deportivas.

- Matadero.

- Protección del Medio ambiente.

Pero, como es sabido, las competencias locales propias no acaban aquí, ya que el legislador sectorial, sea autonómico o estatal, está obligado, en todo caso, a atribuir a los Municipios competencias sobre:

- Ordenación, gestión, ejecución y disciplina urbanística.

- Promoción y gestión de viviendas.

- Conservación de caminos y vías rurales.

- Patrimonio histórico-artístico.

- Atención primaria a la salud.

- Servicios de promoción y reinserción social.

- Suministro de alumbrado público.

- Tratamiento de aguas residuales.

- Transporte público de viajeros.

- Actividades culturales y deportivas.

- Ocupación del tiempo libre.

- Turismo.

- Creación, construcción y sostenimiento de los centros docentes privados.

En relación a las competencias municipales sobre todos estos sectores las Diputaciones desarrollan los instrumentos derivados de su posición institucional de garantía de los principios de solidaridad y equilibrio intermunicipales, así como la prestación integral y adecuada de los servicios derivados de la competencia municipal. Además, debe recordarse que el articulo 36, permite a la Diputación actuar en favor del fomento de los intereses peculiares de la Provincia en función de lo cual, como apunta MORELL "las Diputaciones han encontrado un campo amplio de actividad, supliendo los vacíos de la acción estatal en los campos cultural, social y económico".

Debe tenerse presente que, sobre todos estos sectores materiales las Diputaciones ejercitan funciones concebidas como competencias propias y como tales, presididas por su posición institucional de entes dotados de autonomía y sobre las cuales juega el principio de la garantía institucional. Es evidente que el alcance de dicha garantía en cuanto a la intensidad de la intervención provincial puede variar en función de la voluntad política del legislador sectorial. Pero lo que éste no puede hacer es desconocer que en todos los sectores materiales 
en los que existan competencias municipales propias, las Diputaciones han de desarrollar también como competencia propia, funciones de coordinación cooperación y prestación de servicios supramunicipales. A ello se añade que el legislador de la garantía institucional ha establecido en el articulo 2 LRBRL unos criterios que aún actuando como conceptos juridicos indeterminados limitan la libertad del legislador sectorial en cuanto le indican que la atribución de competencias locales debe realizarse "en atención a las características de la actividad pública de que se trate y a la capacidad de gestión de la Entidad Local, de conformidad con los principios de descentralización y de máxima proximidad de la gestión administrativa a los ciudadanos".

Sin embargo, el hecho de que los ámbitos materiales de las competencias autonómicas sean coincidentes con los ámbitos materiales donde los entes locales han de desarrollar sus propias competencias, ha provocado que las posibilidades reales de que las Diputaciones Provinciales desplieguen su papel institucional se vean seriamente cuestionadas por tres factores principales. En primer lugar, por la aparición de una legislación autonómica que prevé una transferencia de competencias y recursos económicos de las Diputaciones a las comunidades Autónomas. En segundo término, por una interpretación extensiva de la técnica de la coordinación competencial de las comunidades Autónomas respecto de las competencias provinciales. Por último, por el desconocimiento de los instrumentos estatutarios de delegación de competencias autonómicas en las Diputaciones.

\section{EL TRASPASO DE COMPETENCIAS Y TRASFERENCIA DE RECURSOS PROVINCIALES A LAS COMUNIDADES AUTONOMAS.}

No cabe duda que la aparición de las Comunidades Autónomas en el ordenamiento constitucional español ha supuesto la necesidad de producir una alteración en el reparto competencial de los diferentes entes territoriales no sólo en relación al Estado, sino también a las Corporaciones Locales.

Un primer intento de esta reordenación competencial se produjo ya mediante la aprobación de la Ley 6/1980 de 7 de diciembre de Transferencia Urgente y Plena de las Diputaciones catalanas a la Generalidad. En sus artículos 1 al 6 se transferian a la Generalidad tanto las competencias propias de las Diputaciones Provinciales como las que hubiesen asumido por delegación, así como los servicios provinciales las competencias financieras y sus recursos y los propios funcionarios. Contra esta Ley se interpuso en su día recurso de 
inconstitucionalidad por el Gobierno, por vulnerar, en lo que aquí nos interesa las bases del Estado sobre el régimen juridico de la Administración local y la autonomía de la Provincia como ente local, al vaciarla de todas sus competencias, propias y delegadas y de su Hacienda. El Tribunal Constitucional (TC), en su conocida sentencia de 28 de julio de 1981 ,dictó una nutrida doctrina que podemos resumir en los postulados siguientes:

- La Provincia como entidad local es una institución que goza constitucionalmente de una garantía institucional.

- Dicha garantía supone un límite para el legislador ordinario.

- Son contenido esencial de la garantía institucional de la Provincia como ente local su posición de autonomía frente al resto de los entes territoriales en la gestión de sus intereses y el derecho, como tal entidad autonómica que participa de la distribución vertical del poder público, a participar, mediante potestades decisorias, en el gobierno y administración de cuantos asuntos le atañen.

- Corresponde al legislador estatal, a la hora de regular los principios básicos de organización y competencias locales, fijar el alcance concreto de dicha garantía institucional.

- El legislador autonómico debe respetar dichos principios básicos.

En aplicación a estos postulados, el TC entendió contrario a la Constitución el desapoderamiento competencial de las Diputaciones catalanas. De las consideraciones del TC referidas al tema de las competencias, se desprende lo siguiente:

- No se compadece con la autonomía provincial que sus órganos de representación sólo posean competencias de estudio y propuesta.

- El ejercicio desconcentrado de las competencias de una Comunidad Autónoma no puede ser sustitutivo de su gestión en régimen de autonomía.

- Vulnera las bases estatales existentes en ese momento el no tener en cuenta la atribución de competencias para la gestión de los intereses peculiares de la Provincia, asi como de la exigencia de la prestación de unos servicios mínimos.

- Vulnera las bases estatales el desconocimiento de la atribución de potestades decisorias $y$, en concreto, la aprobación de sus propios presupuestos, que es considerada una potestad decisoria fundamental.

La LRBRL ha venido posteriormente a desarrollar la garantía institucional $y$, en lo que concierne a los puntos aquí señalados, conviene resaltar que: 
- El articulo 4 dota a las Provincias, como Administraciones Públicas de carácter territorial de una serie de potestades que debe respetar el legislador autonómico.

- Los artículos 2 y 36 en relación con los artículos 25 y 26, dotan a las Diputaciones Provinciales de competencias propias para intervenir en un amplio conjunto de asuntos.

- El articulo 7 exije que las competencias propias se ejerzan en régimen de autonomía y bajo la propia responsabilidad.

- El artículo 112 les atribuye la aprobación de sus propios presupuestos.

Sin embargo, la propia sentencia del TC de 28 de julio de 1981 también señalaba que :

"Algunos de los que hoy son tales (intereses provinciales) podrían ser configurados como intereses infraprovinciales $y$ atribuirse su gestión a entidades de esta naturaleza, y la defensa y cuidado de otros podrá ser atribuida a la Comunidad Autónoma en la que la Provincia se encuentra para ser gestionados por la propia Comunidad".

Por esta brecha se han lanzado algunos legisladores, autonómicos, especialmente el aragonés y el catalán (Ley $8 / 1985$ de 20 de diciembre reguladora de las relaciones entre la Comunidad Autónoma de Aragón y las Diputaciones Provinciales de su territorio; Ley 5/1987 de 4 de abril de régimen provisional de las competencias de las Diputaciones Provinciales de Cataluña; Ley 8/1987 de 15 de abril, Municipal y de Régimen Local de Cataluña) entendiendo que existe un importante conjunto de competencias provinciales, cuya modificación y consiguiente transferencia al nivel autonómico forma parte de las atribuciones legislativas de sus respectivos Parlamentos. El esquema básico de esta legislación consiste en establecer la posibilidad de la transferencia de la titularidad de una competencia provincial al ente autonómico mediante una ley sectorial. Transferencia que se ve acompañada de los recursos económicos que las Diputaciones destinaban a ejercicio de dicha competencia que son calculados mediante el establecimiento de un porcentaje de participación en los ingresos provinciales.

Estas leyes sectoriales no se han hecho esperar, constituyendo, en estos momentos la amenaza más seria a la propia supervivencia de la entidad provincial, por cuanto suponen el desapoderamiento económico de sus recursos fundamentales. Así, en Aragón encontramos la Ley 4/1987 de 25 de marzo, de Ordenación de la Acción social; la Ley 2/1989 de 21 de abril del Servicio Aragonés de Salud; y la Ley 10/1989 de 14 de diciembre de Protección de Menores. Por su parte, Cataluña ha aprobado la Ley $8 / 1988$ de 7 de abril del Deporte y la reciente Ley $17 / 1990$ de 2 de noviembre de Museos. 
Sin embargo, existen serias dudas de la constitucionalidad de todo este paquete legislativo autonómico. En efecto, el sistema competencial establecido en la LRBRL con relación a las Diputaciones no tiene nada que ver con el establecido en la anterior Ley de Régimen Local de 1955. En esta Ley se establecía un amplio elenco de materias objeto de la competencia de las Diputaciones, aunque luego dichas competencias podían ser perfectamente desconocidas por el legislador sectorial, entonces únicamente estatal, incluso mediante norma reglamentaria, como puso de manifiesto SALAS.

En la LRBRL, en cambio, el legislador estatal básico es, al mismo tiempo, el legislador de la garantía institucional de la Provincia como ente territorial dotado de autonomía. Dicho legislador ha partido, como se recordará, de la definición constitucional de la Provincia como "agrupación de Municipios" y le ha hecho ocupar un papel institucional clave para la garantía de la autonomía local tal como queda definido en el juego combinado de los artículos 31 y 36 de la propia LRBRL. El resultado de esta combinación nos lleva a la constatación de que las competencias propias que la LRBRL atribuye a las Diputaciones son competencias instrumentales que amparan el desarrollo de una función de colaboración y apoyo al ejercicio de las competencias municipales. No hay en el LRBRL una atribución directa de competencias materiales a las Diputaciones, se trata de competencias funcionales que cobran su dimensión material en relación a las competencias propias de los Municipios.

Quisiera insistir en este punto porque de su correcta comprensión depende el futuro institucional de los entes provinciales. La LRBRL cumple la tarea descentralizadora de regular el ámbito de poder local que es indisponible para el legislador sectorial. Para ello, declara un conjunto de servicios mínimos de prestación municipal y un listado de materias sobre las cuales el legislador sectorial debe atribuir a los Municipios algún tipo de competencia. Teniendo en cuenta, además, la debilidad técnica y financiera de muchos de nuestros Municipios, en garantía del propio poder local, encomienda a las Provincias una función de apoyo, reiterada en varios de sus preceptos, para la efectiva realización de los servicios y las competencias que se derivan de la garantía institucional de los entes locales como entidades territoriales dotadas de autonomía.

Esta función de apoyo se expresa en las competencias propias de las Diputaciones que antes se ha expuesto y que, como se puede observar, no tienen contenido material en si mismas. Ciertamente, el legislador estatal básico ha dejado la puerta abierta para que los legisladores sectoriales puedan atribuir directamente a las Diputaciones competencias propias sobre sectores materiales, pero esto ya no es decisivo dentro del modelo de poder local diseñado en la LRBRL. 
Mas decisivo es, por contra, la posición ordinamental que el legislador de la garantía institucional tiene con relación al legislador sectorial, especialmente cuando éste es el legislador autonómico. Como señala en su Exposición de Motivos:

"El mecanismo de cierre lo proporciona, de un lado, la imposición a la legislación sectorial -desde la especial posición ordinamental que a la Ley de régimen local le es propia según ya se ha hecho notar- de la ponderación del expresado criterio (el de la participación en los asuntos públicos) y, de otro, la articulación de las competencias administrativas en la materia de que se trata de forma consecuente con la misma, así como la atribución a la legislación básica estatal de una función de aseguramiento de un mínimo competencial a la Administración local"

Esta posición ordinamental de la LRBRL, tanto como legislador de la garantía institucional, como de legislador básico del régimen local, que ha sido ratificada por el TC en su sentencia de 26 de febrero de 1987, hace que la caracterización institucional de la Provincia, garantizada por el legislador estatal, como ente de apoyo a la autonomia municipal, así como el ámbito instrumental de las competencias propias de las Diputaciones, no pueda ser alterado ni modificado por el legislador autonómico.

El sistema competencial que hemos descrito encuentra una adecuada correspondencia en el régimen de financiación de las Provincias. En efecto, si partimos del hecho de que las Diputaciones no tienen atribuidas directamente por el legislador básico la prestación de servicios públicos concretos, quiere decir que la previsión del gasto de los entes provinciales entra de lleno dentro del concepto de gastos indivisibles. Dicho de otra forma, en la medida que el servicio público derivado de una competencia material se sufraga mediante una tasa o un precio publico, si este servicio se transfiere, la financiación de dicha transferencia se corresponde con el producto que obtiene de la propia tasa o del precio público el nuevo ente que preste el servicio. Sin embargo, en la medida que las Diputaciones no poseen servicios derivados de competencias materiales propias, su actividad se realiza en función de una previa decisión de política presupuestaria que debe concretarse en cada anualidad en relación a los sectores materiales de ámbito municipal hacia los cuales quiere dirigir su apoyo instrumental. El gasto público provincial no está ligado, así , a priori , a ninguna actividad derivada de una competencia sobre una materia concreta.

En correspondencia con ello, el importe de la participación de las Provincias en los Tributos del Estado se distribuye en cada Ley de Presupuestos con arreglo a los criterios del artículo 126 de la Ley 
Reguladora de las Haciendas Locales (LHL). Estos criterios, como es sabido, son:

A)El número de habitantes de derecho de la respectiva Provincia o Isla, según los últimos Padrones Municipales oficialmente aprobados.

B) La superficie.

C) Numero de habitantes de derecho de los municipios menores de $\mathbf{2 0 . 0 0 0 ~ h a b i t a n t e s ~ e n ~ r e l a c i o ́ n ~ a l ~ t o t a l ~ d e ~ h a b i t a n t e s ~ d e ~ l a ~ P r o - ~}$ vincia o Isla".

D) La inversa de la renta per cápita.

E) Otros criterios que se estimen procedentes.

Como se puede observar, estos criterios son acordes al papel institucional de instrumento de reequilibrio territorial que debe cumplir el ente provincial, sin estar ligada previamente su financiación a ninguna actividad material concreta. Esto mismo se pone de manifiesto de la lectura, a sensu contrario, del artículo 127 LHL el cual establece:

"Cuando una Provincia con la utilización de los recursos financieros regulados en la presente Ley, no pudiera ejercer adecuadamente las competencias a que se refieren las letras a) b) c) y d) del apartado 1 del articulo 36 de la Ley 7/1985 de 2 de abril, los Presupuestos Generales del Estado podrán establecer, con especificación de su destino y distribución una asignación complementaria, cuya finalidad será la de cubrir insuficiencias financieras manifiestas".

Las transferencias derivadas de los Presupuestos Generales del Estado están ligadas, por tanto, a la función instrumental de las Diputaciones establecida en los distintos apartados del articulo 36 LRBRL $y$, sólo cuando se produzca una insuficiencia financiera, se destinarán partidas presupuestarias directamente afectadas a un servicio o actividad concreta.

En definitiva, la LRBRL atribuye a las Diputaciones un conjunto de competencias propias que tienen en cuenta la posición institucional de la Provincia dentro del poder local, de forma que tales competencias se configuran como competencias funcionales, con posibilidad de ser dirigidas en apoyo de las distintas necesidades de asistencia de los Municipios para el ejercicio de sus competencias propias, aseguradas, a su vez, por la garantía institucional de los entes locales.

Para ello, sólo cabe predisponer un sistema de financiación dirigido a la suficiencia de dicha posición institucional, sistema que, por tanto, no esta ligado en su esencia a un bloque competencias concreto de materias predeterminadas, sino que tiene como objetivo el posibilitar una determinada capacidad de gasto acorde con las finalidades 
institucionales del ente provincial, de forma que, a través del ejercicio de su autonomía presupuestaria, se concretan en cada anualidad los sectores hacia los que dirigir su colaboración técnica y financiera. Solo un planteamiento de este tipo, en el que se configura un sistema de financiación global, puede hacer realidad el principio constitucional de autonomía local. Si la suficiencia financiera de los entes locales, proclamada en el articulo 142 de la Constitución, se dirigiera individualmente a cada competencia de cada ente local, se estaria falseando el propio principio de autonomía local. Es el conjunto de los poderes locales, tal como aparece garantizado en la LRBRL, el que debe contar con un sistema de suficiencia financiera.

Ahora bien, hay que tener siempre presente que el sistema de financiación de los entes locales es diferente del propio de las Comunidades Autónomas. Esta diferencia se concreta en el reconocimiento de la "autonomía financiera para el desarrollo y ejecución de sus competencias" según establece el artículo 156 de la Constitución con relación a las Comunidades Autónomas, mientras que respecto de los entes locales se establece, en el artículo 143 del texto constitucional, que éstos "deberán disponer de los medios suficientes para el desempeño de las funciones que la ley atribuye a las Corporaciones respectivas". La diferencia no sólo estriba en el distinto tratamiento conceptual de los principios de autonomía y de suficiencia financiera, sino que hay una estrecha ligazón en el primer caso, entre financiación y competencias, mientras que en el segundo, la relación se establece entre financiación y funciones.

Indudablemente, es un régimen mejor para el amparo de la autonomía el establecido con relación a las Comunidades Autónomas. Pero ello no es motivo para desvirtuar el sistema aplicable a los entes locales. Este último depende de dos decisiones del legislador: aquella relativa a las funciones que se reconocen a los entes locales y aquella otra en la que se concretan los medios suficientes para el desempeño de las mismas. Este legislador es tanto el legislador estatal como el legislador autonómico y a ambos les corresponde establecer un equilibrio entre sus propias decisiones que, lógicamente, no puede ser alterado por el otro. Así, el Estado establece, en el ámbito de sus competencias normativas, las funciones que reconoce a los entes locales y una suficiente financiación de las mismas. El legislador autonómico, por su parte, puede reconocer ulteriores funciones y el consiguiente complemento financiero, pero no puede alterar el anterior bloque de decisiones.

Esta doble dependencia del legislador de la competencia y del legislador de la financiación tiene unas consecuencias muy precisas, especialmente para el caso de las competencias provinciales. En efecto, la correspondencia entre el ámbito de actuación del ente local y 
los medios puestos a su disposición no está basado en el coste de la competencia. Este criterio podría ser alegado en último término como vulneración de la garantía institucional. Pero el Sistema establecido en la LHL se basa en dotar a los entes locales de instrumentos que, más allá de los servicios obligatorios, les permiten financiar sus proyectos de actuación y la calidad de los mismos, de forma que se produzca un equilibrio presupuestario. Esto último es paradigmático en el caso de las Diputaciones. Estas se caracterizan por la indeterminación material de sus funciones y por un sistema de financiación basado esencialmente en la participación en los Tributos del Estado. El volumen de esta participación depende de las decisiones que anualmente establezcan los Presupuestos Generales del Estado, decisiones ligadas a la interpretación político institucional que el Estado realice del papel que corresponde a las Diputaciones. Interpretación que no puede ser alterada por el legislador autonómico y que forma parte del carácter bifronte del régimen local en nuestro Ordenamiento. El sistema es, pues, radicalmente distinto al que se produce con relación a las Comunidades Autónomas, tal como lo ha destacado el propio TC en su sentencia de 23 de diciembre de 1982, al tratar de la diferencia entre transferencia de competencias del Estado a las Comunidades Autónomas y a los entes locales, respectivamente :

"El traspaso a las Comunidades Autónomas de tales competencias debe ir acompañado, de otra parte, de los medios financieros que requiere su ejercicio (articulo 150, 2, en tanto que lo que, de acuerdo con el precepto impugnado, la de traspasarse a las Corporaciones locales a las que se transfieren o delegan funciones o servicios son <<créditos presupuestario〉> renovables, por tanto, anualmente".

Así, el volumen de financiación que las Diputaciones reciben del Estado o de sus propios tributos, al estar ligado a las funciones que el propio Estado les ha reconocido, es indisponible por parte del legislador autonómico. A su vez, al ser esta función esencialmente el reconocimiento de una posición institucional de apoyo a la autonomía municipal, de reequilibrio intermunicipal y de fomento de sus intereses propios, corresponde a la autonomía presupuestaria del ente provincial el determinar, en función de una financiación que también tiene un origen de anualidad en el Estado, los sectores de actividad en los que concreta sus prioridades, que no están ligados en modo alguno a materias predeterminadas, sino que pueden sufrir, en función de los propios criterios de orientación política de la Diputación, variaciones en cada ejercicio presupuestario.

En consecuencia, ninguno de los mecanismos establecidos en la legislación sectorial autonómica, relativos a la transferencia de los 
servicios, patrimonio y medios financieros que las Diputaciones pudieran tener destinados a una actividad concreta, pueden ser de aplicación constitucional por la simple razón de que no es posible que se efectúe la transferencia de la competencia provincial, al ser ésta de carácter funcional e indisponible,por aplicación de la garantía institucional, para el legislador autonómico. Por ello, toda ordenación de la transferencia de los medios materiales de una Diputación a la Comunidad Autónoma correspondiente, no puede estar amparada por ningún traspaso competencial y, por tanto, se produciría un puro y simple desapoderamiento patrimonial sin contraprestación alguna, lo que hace que estemos ante un supuesto confiscatorio con tacha de inconstitucionalidad. Cualquier mandato de transferencia de servicios provinciales y de todo su sistema de financiación a una Comunidad Autónoma constituye una vulneración de la autonomía local. Dichos servicios, en la actualidad, están, por definición, ligados a la posición institucional del ente provincial, establecida por el legislador estatal de la garantía institucional, que no puede ser alterada por el legislador autonómico. A su vez, el volumen de recursos económicos que las Diputaciones puedan destinar al mantenimiento de cada uno de dichos servicios, corresponde al ámbito de su autonomía presupuestaria igualmente inatacable por ninguna Comunidad Autónoma.

\section{LA COORDINACION AUTONOMICA DE LAS COMPETENCIAS PROVINCIALES.}

En nuestro ordenamiento jurídico se ha impuesto la concepción funcional de la garantia institucional de los entes locales, de forma que ésta no hace referencia a un listado de competencias exclusivas locales mínimas, que se correspondiesen con intereses exclusivamente locales, en la vieja tradición del "pouvoir municipal ", sino a la garantía de que el ente local pueda intervenir en todos los asuntos públicos que afecten directamente a los intereses de la comunidad política local.

Ya GARCIA DE ENTERRIA afirmó en su día que "no hay la menor base para establecer una partición de esferas entre el Estado y los entes locales que pretendiese apoyarse... sobre una supuesta diversidad natural de las respectivas materias... : las materias propias de una y otra esfera de gobierno son idénticas e intercambiables, como pertenecientes ambas al mismo orden político y suscitadas por la misma naturaleza social del hombre". Este rechazo de la teoría de los intereses naturales de los entes locales ha sido asumida plenamente por nuestro 
TC en su sentencia de 28 de julio de 1981. En efecto, la realidad de la sociedad actual hace que los intereses de los entes locales no sean intereses diferenciados de los del resto de las Administraciones públicas. Por ello, si bien en las competencias propias de los entes locales se debe traducir su posición de autonomía institucional derivada de la definición constitucional de España como Estado políticamente descentralizado, al mismo tiempo, estas competencias se deben coordinar con las de los demás entes públicos por no versar sobre materias de interés exclusivamente local, sino sobre materias en relación a las cuales todos los entes locales pueden ser llamados a intervenir.

A ello se le añade que el concepto de Estado social postula que los intereses de los ciudadanos no sólo queden en el terreno de las espectativas, frente a la actuación de los poderes públicos, sino que reclama la realización material de estos intereses. No hay, pues, una libertad del Estado para actuar o no respecto a las necesidades sociales, sino que nuestra Constitución postula un deber de intervención. El instrumento para esta intervención es, sin duda, la Administración o el conjunto de las Administraciones públicas a las que se reclama que instrumenten remedios frente a las necesidades sociales. Pero no de cualquier forma, sino ateniéndose al principio de eficacia, que pasa a constituir uno de los elementos de la justificación democrática del poder público, bajo el prisma del Estado social. Ahora bien, cuando por efecto del pluralismo político territorial, los intereses sociales se satisfacen a través de una pluralidad de Administraciones, no deja de pesar sobre éstas el principio de mayor eficacia en su actuación conjunta. En el ámbito de la organización, tal como ha ratificado el TC (STC 27/87 de 27 de febrero), el principio de coordinación legitima al Estado para, en orden al buen funcionamiento global de las Administraciones públicas, imponer a todas ellas la existencia de elementos organizativos que lo hagan posible, asi como un tipo de procedimiento de actuación. Así, pues, todos los medios formales de coordinación se justifican por el principio de legitimación democrática del Estado, de la eficacia de su funcionamiento conjunto.

No ocurre lo mismo, en cambio, cuando se trata de aplicar el principio de coordinación a las competencias sectoriales. Aquí el principio que preside la competencia de coordinación es el del respeto a la función que el principio de pluralismo político atribuye a cada ente territorial. Desde el momento que le corresponde a la instancia superior formular los intereses generales el principio de coordinación, en los sectores materiales afectados exige de las instancias inferiores un respeto a su efectiva realización. Por ello, los objetivos señalados en las políticas generales, no pueden ser entorpecidos por otros objetivos parciales contradictorios como el objetivo global. De algún modo 
estariamos ante una manifestación del principio de lealtad constitucional que no implica una subordinación activa a la política general sino una abstención de realización de políticas que impidan la consecución del objetivo general. La coordinación competencial opera, de este modo, como un limite a la plena disposición de la competencia por parte del ente sometido a coordinación. Este, en base al principio de autonomía, no esta obligado a colaborar en dicha política, pero si a no entorpecerla practicando políticas distorsionantes. Igualmente, es requisito necesario para la coordinación la formulación previa, por el ente en posición de supremacia, de los objetivos de política sectorial que pretende que sean respetados.

La LRBRL recoge como no podía ser menos el principio de coordinación de las competencias de los entes locales con las de las demás Administraciones públicas (art. 7, 2). Principio que se desarrolla en el articulo 10 con carácter general, y en el articulo 36 en lo que se refiere a la coordinación de los planes provinciales de las Diputaciones por parte de las Comunidades Autónomas.

Sin embargo, ya en el propio articulo 10 LRBRL se señala que "las funciones de coordinación no afectarán en ningún caso a la autonomía de las Entidades locales". Esta advertencia legal de que la coordinación no puede eliminar la autonomía local, debe entenderse referida, en primer lugar al hecho de que el ejercicio de la coordinación no puede suponer la pérdida de las potestades ordinamentales de los entes locales previstas en el articulo 4 LRBRL (potestad reglamentaria $y$ de autoorganización; potestad tributaria y financiera; potestad de programación y planificación; etc.). En segundo término, si, mediante la coordinación, las competencias locales son ordenadas a través de órganos de relación o en un procedimiento complejo, éstas no pueden perder, en ningún caso, sus caracteres de competencias exclusivas y decisorias. Por último, el respeto a su posición de autonomía hace que los entes locales coordinados no tengan que participar necesaria y activamente en los objetivos diseñados por las Administraciones ordinamentalmente superiores.

En lo que se refiere a la coordinación de planes provinciales, el articulo 36 , en su apartado 2 ,a) remite a un procedimiento de coordinación previsto en el articulo 59 de la propia LRBRL. Este precepto trata de armonizar la posición ordinamental de los éntes locales como entes dotados de autonomía, con la necesidad de una actuación coordinada del conjunto de las Administraciones públicas. Así, en primer lugar establece la regla de la proporcionalidad de los medios a los fines perseguidos en la utilización de la técnica de coordinación competencial, imponiendo la necesidad de haber agotado otros sistemas de coordinación menos gravosos para la autonomía del ente local establecidos 
en los artículos 53,57 y 58 LRBRL y que constituyen técnicas de coordinación organizativa. Ahora bien, además de lo anterior, el articulo 59 exige que la coordinación competencial de sectores materiales se instrumente con arreglo a requisitos bien determinados.

Así, la facultad de coordinar la actividad de los entes locales ha de ejercitarse mediante Ley. Ley que no puede contener una definición genérica de la actividad a coordinar, sino, al contrario, debe definir el interés general superior del ente prevalente de forma concreta y en relación con una materia, servicio o competencia. En segundo término, la Ley debe contener un único instrumento: el plan sectorial, que, a su vez, viene delimitado por nuevos requisitos en cuanto a su contenido y forma de elaboración. En lo referido a los requisitos de contenido, éstos son dos. La fijación de un objetivo en relación a la materia servicio o competencia declarada de interés superior y la determinación de prioridades para la consecución de dicho objetivo. Por su parte, la forma de elaboración del plan está sometida al articulo 58, 2. LRBRL, que exige una participación del ente local afectado, bien en el órgano que lleve a cabo el plan, bien en la fase de elaboración del mismo, de forma que se consiga una armonización de los intereses públicos afectados. Por último, el plan no puede ocupar todo el campo de la actividad. La LRBRL entiende el plan como un documento de instrucciones entre objetivos y prioridades, que fije el marco donde deben actuarse las competencias de los entes locales.

En definitiva, si bien la LRBRL atribuye a las Diputaciones, como competencia propia, la aprobación anual de un plan provincial de obras y servicios, esta competencia está condicionada por la facultad que ostentan las Comunidades Autónomas de asegurar, en sus respectivos territorios, la coordinación de los diversos planes provinciales. El objeto de la facultad autonómica estriba en condicionar el ejercicio de la competencia provincial al objetivo autonómico prefijado mediante Ley en un Plan, aunque sea la Diputación quien disponga, dentro de ese marco, de todas sus competencias. Así, si un plan autonómico fija como objetivo de su política de obras públicas, un programa de reparación y acondicionamiento de carreteras, la Diputación no está obligada a dotar en sus presupuestos una partida destinada a tal fin pues corresponde plenamente a su autonomía la fijación de éstos. Pero, si presupuesta recursos para el arreglo de carreteras, dichas partidas deben ajustarse a las prioridades del plan autonómico, aunque sea la Diputación quien también, finalmente, realice directamente el gasto y la ejecución de la obra.

No podemos ignorar, sin embargo, que el TC, en la sentencia de 27 de febrero de 1987, relativa a la ley valenciana 2/83 de declaración de interés general autonómico de determinadas funciones propias de 
las Diputaciones Provinciales, ha introducido alguna flexibilidad a este sistema. En primer lugar, ha admitido, como indicadores de la afectación de las funciones provinciales a las competencias autonómicas, el efecto extraprovincial de la actividad y la afectación de los servicios o competencias autonómicas. A mi juicio, la constitucionalidad de estos indicadores es más que discutible, especialmente el constituido por "la afectación de los servicios o competencias autonómicos", el cual no es un indicador en sí, sino precisamente el objeto que debe ser medido por indicadores. En mi opinión, el grado de afectación de los niveles autonómicos debe concretarse mediante indicadores de tipo demográfico, de condición social calidad, de vida etc.

Una segunda flexibilización del sistema de la LRBRL se produce cuando el TC considera conforme al bloque de constitucionalidad la remisión que el articulo 4 de la Ley valenciana hace al Consejo Ejecutivo de la Generalidad para que sea él quien fije por Decreto las directrices de los planes de coordinación sectorial. A mi entender, en este extremo no se cumple con la reserva de Ley que establece el articulo 59, 2 LRBRL, al ser cada directriz concreta la que establezca los limites a las competencias de las Diputaciones, las cuales -como señala....el magistrado RUBIO LLORENTE en voto particular- al haber sido otorgadas mediante Ley, sólo por Ley, tal como establece el articulo 59, 2 LRBRL, puede limitarse o condicionarse su ejercicio.

Con todo, también en la propia sentencia del TC encontramos alguna declaración de sumo interés con relación a la posición de autonomía de las Diputaciones respecto a las directrices autonómicas de coordinación competencial. Así, el TC ha señalado expresamente que las directrices "no pueden suponer una modalidad de control, ni colocar a las Diputaciones Provinciales en una posición de subordinación jerárquica o cuasijerarquica, incompatible con su autonomía". Más adelante, subraya que la coordinación "no puede traducirse en la emanación de ordenes concretas que prefiguren exhaustivamente el contenido de la actividad del ente coordinado, agotando su propio ámbito de decisión autónoma; en cualquier caso, los medios y técnicas de coordinación deben respetar un margen de libre decisión o de discrecionalidad en favor de las administraciones sujetas a la misma, sin el cual no puede existir verdadera autonomía". También hay que destacar la declaración de inconstitucionalidad de la técnica de suspensión gubernativa de los actos que contradigan las directrices de coordinación, por vulneración del sistema general de control de la legalidad de los actos de los entes locales establecido en la LRBRL. Finalmente, se puede recordar que, en lo que se refiere a la posibilidad de suspender las subvenciones autonómicas a aqueIlas Diputaciones que incumplan las directrices, el TC ha declarado 
que dicha suspensión solo puede referirse a aquellas subvenciones relativas a las materias concretas respecto de las cuales se hayan incumplido las directrices de coordinación.

\section{LA DELEGACION DE COMPETENCIAS AUTONOMICAS EN LAS DIPUTACIONES PROVINCIALES.}

La utilización de los aparatos administrativos de las Diputaciones Provinciales para la puesta en marcha del proceso de descentralización política surgido con el modelo autonómico de la Constitución de 1978, es todavía un capitulo que queda por escribir en nuestra breve pero intensa vida institucional e, incluso, no es aventurado decir que, de escribirse, finalmente lo será cuando haya transcurrido un periodo más dilatado de vida democrática. Mi la racionalidad doctrinal, ni el impulso político han dado todavia frutos apreciables. En todo este proceso nos encontramos, en efecto, con una tensión permanente. De un lado, se inscribe un discurso autonomista frente al Estado, reclamando la descentralización. De otro, se practica una política de centralización a nivel autonómico. Esta tensión es comprensible en la etapa de implantación de las instituciones autonómicas. En primer lugar, nos encontramos con el hecho de que la introducción de las Comunidades Autónomas como ente territorial, que se interpone entre el Estado y las Corporaciones locales, ha hecho derivar un pulso político hacia el Estado en el caso de aquellas Comunidades Autónomas de mayor identidad política y cultural, pero, en la mayoría de los casos, el pulso se va a jugar en el terreno de la gestión del territorio y con relación a las Corporaciones locales.

A este dato hay que ligar el hecho de que las respectivas clases políticas autonómicas tienen mayor dificultad de enfrentarse con el aparato del partido político que gestiona en el nivel estatal, que con el aparato que gestiona los intereses locales. Téngase en cuenta que, al estar la mayoría de los partidos organizados a nivel regional, el aparato local depende de esta organización regional. Por ello, es posible pensar que el desarrollo bifronte del ordenamiento local en España tiene alguna relación con la mutua complementariedad entre el aparato estatal y el local para actuar de contrapeso al aparato regional. También para el caso de los partidos regionalistas o nacionalistas rige esta regla, pues el partido regional tiene como lógica la hegemonía en su territorio, desplazando del poder local a los partidos de implantación nacional, los cuales, a su vez, utilizan su poder local como contrapeso a la ideología nacionalista o regionalista. 
A ello se une el efecto de la pluralidad de instancias de poder territorial, que ha configurado mapas políticos en los cuales, en pocas ocasiones, la titularidad de los gobiernos regionales se complementa con la titularidad de todas las Diputaciones Provinciales de la Comunidad Autónoma. Por ello, no es fácil pensar en la implantación de mecanismos de administración indirecta, cuando aún la propia comunidad Autónoma no estuviese asentada como institución política, ya que este asentamiento no puede prescindir, en el terreno de la realidad política, de la presencia directa en territorio de la propia Administración autonómica, como referente de poder político directo ante los ciudadanos.

Frente a esta realidad se han enfrentado, en vano, otra serie de argumentos, pese a su valor como elementos de configuración de un Estado que, además de descentralizado, debe ser un Estado Social, Democrático y de Derecho. Estos tres argumentos no son otros que el de la racionalización de medios, el de la profundización de la descentralización y el de la obtención de un resultado eficaz en la gestión pública. La racionalización de los medios fue uno de los principales argumentos utilizados en el Informe de la Comisión de Expertos sobre Autonomias. Para dicha Comisión, "mas severas y decididas deben ser las previsiones tendentes a evitar la burocratización de las Comunidades Autónomas. La formación de un aparato administrativo extenso debe evitarse tanto en los niveles centrales como periféricos". Por lo que respecta a la Administración periférica de las Comunidades Autónomas, se afirmaba tajantemente : "su creación misma no debe llegar a producirse en ningún caso". En consecuencia, la Comisión concluía: "Las Diputaciones deben quedar convertidas en el escalón administrativo intrarregional básico: es preciso fortalecer sus servicios, dotarlas mejor, integrar en su organización los servicios periféricos de que se ha de desprender la Administración del Estado para que puedan asumir el ejercicio de competencias por transferencia o delegación de las Comunidades Autónomas y atender ordinariamente la prestación de los servicios que están encomendados a la gestión regional".

Desde la perspectiva de la profundización en la descentralización, opera un doble mecanismo. De una parte, el grado de autonomía política de las Comunidades Autónomas debería jugar en favor de un aumento del poder de autogobierno, frente al tradicional modelo de centralización estatalista. Pero, de otra parte, este poder de autogobierno, desde una óptica de profundización democrática, debía haber puesto como eje de la descentralización a aquellos entes públicos territoriales más idóneos para propiciar la participación ciudadana en la gestión de los asuntos públicos. Por ello, no debe extrañar que se miren con recelo aquellas propuestas de "interiorización" del régimen 
local en el ámbito competencial de las Comunidades Autónomas cuando todos los signos indican una fuerte tendencia a la centralización regional. Tal como señala GARCIA DE ENTERRIA, “lo que está prevaleciendo en la práctica es que las Comunidades Autónomas han montado ya o están montando apresuradamente su propia red de agentes periféricos, que resulten más comodos de manejar que la utilización de una entidad intermedia como la Diputación, representativa y autónoma".

Finalmente hay que destacar el profundo cambio que ha producido el reconocimiento constitucional de los derechos prestacionales en la concepción de las relaciones entre los ciudadanos y las Administraciones públicas. El individuo se sitúa ahora, en muchas ocasiones, junto a la actividad administrativa, colaborando con el la para facilitar la obtención de sus beneficios colectivos. El hecho de que en la generalidad de los casos las distintas Administraciones actúen sobre una misma materia o sector de actividad debe extremar, desde la perspectiva del resultado social que se deriva de su actuación global aquellas técnicas de relación que eviten las interferencias y aseguren la eficacia. Como señala PAREJO, este principio de eficacia "implica -desde la perspectiva, única válida, del ciudadano, de la colectividad administrativa- una condición de calidad, en sentido de economicidad, utilidad $y$, en suma, de bondad de la actuación en su resultado". Dicho principio de eficacia, pues, debe predicarse, desde la perspectiva del Estado social a la intervención conjunta de las Administraciones que comparten las decisiones y la responsabilidad en la prestación de los servicios públicos.

Junto a la bondad de los argumentos señalados, lo que hay que destacar es que el principio de administración indirecta fue uno de los puntos de los Acuerdos Autonómicos de 1981 entre el Gobierno de la UCD y la dirección del PSOE. Dichos Acuerdos tuvieron su reflejo en los artículos 11 a 17 de la LOAPA, convertidos luego, tras la sentencia del TC de 5 de agosto de 1983, en los artículos 5 a 11 de la Ley del Proceso Autonómico, debiendo señalarse que ninguna de las impugnaciones relativas a estos preceptos tuvieron acogida en las resoluciones del TC. Estos acuerdo políticos tuvieron, además, su reflejo en una serie de Estatutos de Autonomía (Andalucía, Valencia, Aragón, Castilla-La Mancha, Extremadura y Castilla y León) que, salvo algunas especificaciones, transcribian el último apartado del punto 5. 1 de los mencionados Acuerdos Autonómicos:

"La Comunidad Autónoma podrá transferir o delegar en las Diputaciones, mediante Ley aprobada por mayoría absoluta, facultades correspondientes a materias de su competencia. Esta Ley preverá en cada caso la correspondiente transferencia de 
medios financieros, así como la forma de dirección y control que se reserve la Comunidad".

La puesta en práctica, sin embargo, de estas previsiones sobre delegación de competencias, ha sido muy limitada. Sólo en tres Comunidades Autónomas hay una legislación de desarrollo del régimen estatutario de delegaciones -Andalucia, Aragón y Castilla y León- y de éstas sólo en dos se producen delegaciones expresas, en Andalucía en materia de carreteras y servicios sociales (Ley 11/1987 de 26 de diciembre) y en Aragón en materia de abastecimiento y distribución de aguas y redes de saneamiento (Ley $8 / 1985$ de 20 de diciembre), siendo la de Castilla y León una norma de desarrollo procedimental (Ley 6/1986 de 6 de juniol.

Igual ha ocurrido con la figura de la gestión ordinaria de servicios, también producto de los Acuerdos Autonómicos, y recogida en los Estatutos de Autonomía antes mencionados. Quizá, el último eslabón de esta cadena de arrinconamiento político de la figura de la gestión ordinaria de servicios lo constituye la Ley 11/1987, reguladora de las relaciones entre la comunidad de Andalucia y las Diputaciones Provinciales de su territorio, en la cual no aparece ya, ni siquiera, mencionada dicha figura de administración indirecta.

Esta tendencia a olvidar la posibilidad de actuar a través de una administración indirecta, es a mi juicio profundamente desacertada en la actualidad. Si partimos de considerar ya asentadas institucionalmente a las Comunidades Autónomas, lo que procede por parte de éstas es que inicien un proceso de verdadera "interiorización" de los entes locales de su territorio. Pero esta interiorización no debe suponer, como hasta ahora, un intento de fagocitación, pues, en tal caso, los entes locales reivindicarán el estar lo más lejano posible del poder autonómico y seguirán llamando en su ayuda al poder estatal. Afirmada ya la representatividad política de las instituciones autonómicas, no tiene sentido pretender que se es la única institución relevante en todo el territorio autonómico, pues el lo supone, entre otras cosas, un desconocimiento del modelo constitucional y de la raíz profunda de nuestra historia democrática asentada en las elecciones locales. El fracaso de la implantación de un sistema de administración indirecta no ayuda en nada a la legitimación de las Comunidades Autónomas en sus reivindicaciones frente al Estado. Si es cierto que un Estado autonómico niega la concentración del poder en manos de las Instituciones generales, un Estado Social y Democrático de Derecho, también niega la concentración del poder territorial en las Instituciones autonómicas. La estructura de nuestra administración local requiere que la tendencia, generalizada en el plano de los principios, hacia la municipalización de servicios solo es realizable, desde la óptica 
de la autonomía local, bien con una profunda remodelación de la dimensión de nuestros actuales Municipios, bien con el concurso de los entes territoriales locales supramunicipales. Posiblemente, en este esfuerzo habrá que cambiar algunas cosas -muchas o pocas según los casos- del funcionamiento de las Diputaciones Provinciales, pero de su eliminación funcional o institucional, con el consiguiente único contacto entre Municipio y comunidad Autónoma, solo se derivaría una paulatina y segura desaparición del poder local en España. 
